This item was submitted to Loughborough's Research Repository by the author.

Items in Figshare are protected by copyright, with all rights reserved, unless otherwise indicated.

\title{
Effects of different chair-based exercises on salivary biomarkers and functional autonomy in institutionalized older women
}

PLEASE CITE THE PUBLISHED VERSION

https://doi.org/10.1080/02701367.2018.1563272

\section{PUBLISHER}

(c) 2019 SHAPE America. Published by Taylor and Francis

\section{VERSION}

AM (Accepted Manuscript)

\section{PUBLISHER STATEMENT}

This is an Accepted Manuscript of an article published by Taylor \& Francis in Research Quarterly for Exercise and Sport on 5 February 2019, available online: http://www.tandfonline.com/10.1080/02701367.2018.1563272.

\section{LICENCE}

CC BY-NC-ND 4.0

\section{REPOSITORY RECORD}

Rieping, Tais, Guilherme Eustaquio Furtado, Rubens Vinicius Letieri, Matheus Uba Chupel, Juan C. Colado, Eef Hogervorst, Edith Filaire, Ana Maria Teixeira, and Jose Pedro Ferreira. 2019. "Effects of Different Chairbased Exercises on Salivary Biomarkers and Functional Autonomy in Institutionalized Older Women". figshare. https://hdl.handle.net/2134/36933. 
WOMEN

2 Purpose: The aim of this study was to test the effects of chair-based exercise programs on salivary stress hormones, physical fitness and functional autonomy of institutionalized older women. Methods: A total of 47 participants $(80 \pm 8.04$ years old $)$ were recruited and allocated into three groups: chair based aerobic exercises (CAE, $n=19)$, chair-based elasticband strength exercises $(\mathrm{CSE}, \mathrm{n}=15)$ and a control group $(\mathrm{CG}, \mathrm{n}=13)$. A 14-week exercise intervention was done for the CAE and CSE, two times per week, in no consecutive days. The CG did not participate in any type of exercise but kept their regular lifestyle. Fear of falling, autonomy, physical fitness, salivary cortisol and alpha-amylase levels were assessed before and after the intervention. Results: CAE improved upper and lower body strength, agility-dynamic balance and autonomy with fear of falling decreasing significantly $(p<.05$, moderate effect size). Both exercise groups showed a trend towards an increase in salivary alpha-amylase levels $(\mathrm{CAE}=43 \%, d=.31$ and $\mathrm{CSE}=44 \%, d=.41)$. Conclusion: Both exercise programs were able to improve functional autonomy, even in elders over 80 years of age. However, it might be interesting to investigate the effectiveness of combining both aerobic and strength exercises in a unique protocol. The modulation effect of exercise in the hormonal responses needs to be further explored.

Keywords: Activities of daily life, alpha-amylase, cortisol, physical fitness, autonomy. 
WOMEN

Aging is a dynamic and complex process that involves factors such as the environment, genetics, and lifestyle (Spirduso, Francis, \& MacRae, 2005). It causes losses in the central nervous, neuromuscular and skeletal systems, as well as deterioration of many physical-functional fitness functions, such as walking speed, posture, balance, agility and ultimately leading to lack of autonomy and institutionalization (Granacher, Lacroix, Muehlbauer, Roettger, \& Gollhofer, 2013). These justify the need to focus on ways to maintain and improve global health and well-being related to physical health in aging populations.

Benefits promoted by regular exercise in the older adults include the reduction of functional limitations, the improvement of functional skills to perform daily life activities, even in people with reduced mobility (Sherrington et al., 2016). Also, fall events in this age group are very common and are clearly related to the loss of muscle function, postural instability, change in gait pattern, poor balance, visual problems and the use of some medications (Burton et al., 2015).

The literature has shown the effectiveness of both aerobic and strength exercises with elastic bands in improving physical fitness in older adults (Granacher et al., 2013). These also have the advantage of being affordable and adaptable for people with reduced mobility (Chen, Li, Chang, Huang, \& Cheng, 2015). However, the types of exercise and the methods used can determine the degree of exercise participation and the results obtained, especially in populations with special needs conditions (Nelson et al., 2007), given that they may be more challenging to motivate in order to join exercise programs (Sequeira, 2007). The Chair-Based Exercise (CBE) protocol was shown to be a suitable and appropriate method of exercise for inactive older adults (Robinson et al., 2014). It consists of structured and progressive 


\section{WOMEN}

exercises performed with a chair for support that guarantees the individual's stability during the session, respecting individual limitations without discouraging individuals to reach beyond their limits (Furtado et al., 2016).

CBE aerobic programs have been shown to improve cardiorespiratory fitness, decrease fear of falling and to have cognitive benefits in mentally healthy older adults (DeSure, Peterson, Gianan, \& Pang, 2013). Recent studies have also demonstrated the effectiveness of exercise programs using elastics resistance exercises. Elastic is an alternative to traditional muscle strength exercise devices which reduces the risk of injury allowing individuals to perform a range of ergonomic movements and adjust the training intensity, eliciting higher intrinsic motivation in healthcare practitioners to advocate strength exercises (Colado \& Triplett, 2008).

Exercises, aimed at both aerobic and muscle-strength development, trigger a series of changes in the autonomic nervous system (ANS) regulated mechanisms. Several studies have revealed that alpha-amylase (AA) levels increase under conditions of physical and psychobiological stress (Urs Markus Nater et al., 2006). It main function is the organic degradation of starch molecules in the digestion process, and it has also been implicated in the modulation of alpha-adrenergic and beta-adrenergic mechanisms (Lehninger, Nelson, \& Cox, 2007; U. M. Nater \& Rohleder, 2009). While vigorous exercise is related with immune suppression and a higher prevalence of upper respiratory tract infections, moderate exercise seems to promote activation of this enzyme at non-pathological levels increasing health benefits (Walsh et al., 2011).

Cortisol (CORT) is another essential hormone and considered to be the main modulator of the adaptive response to biological and psychological stress (Lehninger et al., 2007). CORT is produced by the adrenal glands, involved in energy metabolism, regulation 


\section{WOMEN}

of the immune system and bone metabolism. Its release is the result of nerve stimuli sent by the ANS when facing a stressful situation (Lehninger et al., 2007). CORT has catabolic functions, increases blood pressure, increases blood glucose and energy availability to the muscle, concomitant with a suspension of anabolic activities (Fries, Dettenborn, \& Kirschbaum, 2009).

Unlike AA, CORT has been well studied over many years and there are several studies that report its increased levels with exercise (Mckune, Bach, Semple, \& Dyer, 2014). However, no exercise intervention studies relating AA and CORT concentrations with simultaneously objective and subjective measurements of physical and functional autonomy in older women have been done, with most exercise studies on AA focusing in adults or athletes (Allgrove, Gomes, Hough, \& Gleeson, 2008).

This study aims to inspect the effectiveness of two CBE programs, one aerobic and the other a muscle-strength elastic band program on the behavior of biological stress/autonomous function biochemical markers (AA and CORT), physical fitness and functional autonomy in institutionalized older women.

\section{Methods}

\section{Recruitment}

This study is a chair-based exercise intervention with institutionalized-dwelling older women, part of the XXXXXXXXX study protocol (XXXXXX et al., XXXXX). Participants were older women living in two different home care services (HCS). A presentation was done to all eligible participants and HCS staff, communicating the stages of the study, possible risks involved and the option of leaving the intervention spontaneously. Participants signed an informed consent term. This study was approved by the Ethics and Human Subjects Review 
WOMEN

Board of the XXXXXXXXXXXX XXXXXX XXXXXXXX, University of XXXXXXXX, XXXXXXXX (University of XXXXXX code number CE/XXXXXXXX/000202013), respecting the guidelines for human research of the Helsinki declaration.

\section{Sample Selection Criteria}

Participants had to be over 75 years old and to have their medication treatment stabilized. If there was any uncontrolled comorbidity, the participation in the classes was not permitted. The exclusion criteria included: completing the ' 8 -foot-up and go test' (8-FGT) in more than 30 seconds (revealing of a high mobility dependency); uncontrolled respiratory diseases and musculoskeletal impairments that prevented the performance of the physical fitness tests; cognitive, hearing and visual impairment; severe morbid obesity or the use of drugs that significantly impaired attention; being engaged in an alternative exercise training program.

\section{Sample Size}

Calculation of sample size was done using $G^{*}$ Power (version 3.1.9.2). Alpha was adjusted at .05 and power at .85 for repeated measures ANOVA. This required a sample size of 75 participants. The initial sample was composed of 70 institutionalized sedentary women from two nursing homes. Additionally, 18 participants were recruited in the same centers to prevent an estimated sample loss of $30 \%$ of participants. At the end of the intervention, the final sample was composed of 47 women (age $=80 \pm 8.04$ years old), allocated into three groups: chair aerobic exercises $(\mathrm{CAE}, \mathrm{n}=19)$, chair elastic-band muscle strength exercises $(\mathrm{CSE}, \mathrm{n}=15)$ and a non-exercise control group $(\mathrm{CG}, \mathrm{n}=13)$. A 14-week CBE intervention was applied in the CAE and CSE groups. The CG group did not participate in any type of 


\section{WOMEN}

120

121

122

123

124

125

126

127

128

129

130

131

132

133

134

135

136

137

138

139

140

141

142

exercise intervention but was encouraged to engage in complementary activities provided by the HCS like outside tours, art education and cultural activities (see figure 1).

\section{[Insert figure 1 about here, please]}

Figure 1 - Flowchart of the study design according to CONSORT guidelines for experimental studies.

\section{Outcome Measures and Data Collection}

The data collection was done in the morning period (09:00 to 11:30 am), before and after the 14-weeks intervention. The same variable, for all the participants, was always measured by the same staff of the research team, without making reference to the exercise programs. The functional autonomy was evaluated by psychometric rate scales and the physical- functional fitness tests as described above. The quality of data was analyzed and reported for each physical-functional fitness measure through scores of internal consistency reliability (ICR).

\section{Stress hormones data collections}

Saliva was collected between 09:00 - 11:30h a.m., with a minimum of 30 minutes after first diurnal food intake. Participants were seated, with their head tilted down, eyes open and instructed to perform a minimum of orofacial movements. The saliva was collected into polypropylene tubes, which were sealed and refrigerated immediately after collection of the sample. The levels of salivary cortisol (sCORT) were evaluated by ELISA (Salimetrics, UK), and alpha salivary AA (sAA) by kinetic assay (Salimetrics, UK), following the manufacturer instructions. The detection limits and correlation with serum samples were reported by the manufacturer as $<.007 \mathrm{ug} / \mathrm{dL}, \mathrm{r}=.91$ respectively for sCORT and $1 \mathrm{pg} / \mathrm{mL}, \mathrm{r}=.96$ for sAA. 
WOMEN

\section{Physical-functional fitness screen}

147 The Physical Fitness test battery was used since it is appropriate for research and clinical 148 purposes in older populations (Rikli \& Jones, 2013). Lower-body dynamic muscle strength is determined with the thirty seconds chair-seated and stand (30s-CS) test that assess the total number of chair-stands finished per time $(\mathrm{ICR}=.86)$. The upper body strength is determined with the thirty seconds arm-curl (30s-AC) test that evaluates the total number of arm curls executed in that time $(\mathrm{ICR}=.92)$. The upper-body flexibility was determined with the back scratch test (BST), that considers the distance (centimeters) of overlap or between the tips of the middle fingers of the back $(\mathrm{ICR}=.84)$. The 8 -FGT was used for assessment of dynamic balance, which evaluates the time needed for the participant to get up from the chair around either side of a cone placed at 8 feet distance and to sit back down $(\mathrm{ICR}=.80)$. The physical tests stations were set in circuit-style to minimize fatigue. Participants performed each test two times in order to get familiarized with them.

Self-perceived risk of falls

Falls Efficacy Scale (FES) was used to measure fear of falling. The minimum score is 100 and the maximum score is 10 points. The lower score corresponding to a high self-efficacy and low fear of falling (Tinetti, Richman, \& Powell, 1990);

\section{Instrumental and independent activities of daily living}

The Lawton Scale of Instrumental Activities of Daily Life (IADL) and the Katz Index of 


\section{WOMEN}

performance in the daily activities. In the IADL, a summary score indicates low function, dependent (zero) to high function and independent, 8 points (Sequeira, 2007). In the ADL, A total of 6 points designates full function, 4 points denotes moderate disability, and 2 or less indicates severe functional disability (Wallace \& Shelkey, 2007).

\section{Charlson Comorbidity Index (CCI)}

The severity of comorbidity was assessed according to the CCI, based on the register of diseases or clinical conditions of each individual, based on 19 conditions forming a single and continuous index (Charlson, Szatrowski, Peterson, \& Gold, 1994). In our study sample, there was a prevalence of diagnosed mental disorders, such as depression, anxiety and chronic stress.

\section{Anthropometric assessment}

The standard and described procedures were followed (Chumlea \& Baumgartner, 1989).

Body mass was assessed using a compact scale (Seca ${ }^{\circledR}$, model 770 , Germany) with a precision of 0.1 kilos. Stature was analyzed using a stadiometer (SecaBodymeter ${ }^{\circledR}$, model 208 , Germany) with a precision of 0.1 centimeters. For body mass index (BMI) the standard formula was applied.

\section{Exercise programs}

The two exercise programs were designed and developed by health professionals, based on the American College of Sports Medicine guidelines (Nelson et al., 2007), and CBE protocol and are described in detail below. The sessions of both CBE were executed three times per week, on non-consecutive days (separated by 48 hours), for 14 weeks, and lasted 45 minutes. A total of 35 sessions was offered. An attendance of the exercise classes of $60 \%$ to $70 \%$ was 


\section{WOMEN}

193

194

195

196

197

198

199

200

201

202

203

204

205

206

207

208

209

210

211

212

213

214

215

216

determined as a minimum for each participant to be part of the CBE groups. During the intervention, no participant was excluded from both CBE for consecutive absences or low participation, but they were not considered for statistical analysis.

\section{Chair-aerobic exercises to improve walking (CAE)}

CAE was structured with 5 minutes of warming-up, with six general mobility exercises and dynamic stretching; 35 minutes with 7 to 10 exercises with chair support, using repetitions of callisthenics and body weight exercises aimed to improve walking. The circuit training protocol was used increasing complexity of movements and sequences between series, preserving the aerobic characteristic of the session; 5 minutes of cooling-down with stretching and breathing exercises. Furthermore, heart rate monitors (POLAR, M810) were randomly used in five participants during the exercise sessions, as aid adjustment and control of training loads. For safety reasons, exercise intensity was indirectly predicted using the Karvonen's formula but with maximal heart rate $\left(\mathrm{HR}_{\max }\right)$ being calculated using Franklin formula $\left(\mathrm{HR}_{\max }=207\right.$ beat per minute $-0.7 \mathrm{x}$ chronological age $)$. Additionally, the modified BORG PES was used, comprising a subjective rate scale ranging from 0 to 10 points, with identical intervals and with reference to the quality of effort: (0) nothing at all; (1) very weak; (2) weak; (3) moderate; (4) somewhat strong; (5-6) strong; (7-9) very strong; (10) very, very strong (almost maximal).

\section{Chair elastic-band muscle strength exercises (CSE)}

A periodization CSE program was followed by the participants using the three less resistante elastic bands: yellow, red and green (Thera Band ${ }^{\circledR}$, Akron, Ohio, US), with the support of a chair. Participants performed two sessions of pre-intervention familiarization to adjust to the 


\section{WOMEN}

217 Rated Perceived Exertion (RPE) scale, learn the correct technique of the exercises, select the

218 color, length, and width of the grip of the bands. Each exercise session consisted of (a)

219 general 5-minutes warming-up including six exercises for general body activation; (b)

220 followed by 35 minutes of an elastic-band resistance including 8-10 exercises, 15-20

221 repetitions, 2-3 series and cadence of execution (2 seconds of concentric phase and 2-3

222 seconds of eccentric contraction); (d) completed by 5 minutes of cooling-down with brief

223 static stretching and breathing exercises for the muscles trained. The elastic band resistance

224 exercises were chosen in order to use mainly multi-joint exercises. The intensity was held at

22510 submaximal repetitions with a perceived exertion level of 6 (somewhat-hard) to 8 (hard)

226 on the OMNI scale (Colado \& Triplett, 2008), that comprises a subjective effort scale ranging

227 from 0 to 10 points, with same intervals to the quality of effort: (0) extremely easy; (1-2)

228 easy; (3-5) somewhat easy, (6-7) somewhat-hard; (8) hard; (9-10) extremely-hard (Robertson

229 et al., 2003). The loads were adjusted every week to maintain appropriate training intensities

230 by adapting the levels of resistance.

Statistical Analyses

Descriptive statistics are presented using mean and standard deviations (SD). The normal

distribution was tested using the Shapiro-Wilk test. Data were log transformed to reduce bias arising from non-uniformity. Since some assumptions for repeated measures ANOVA performance were violated, we used univariate analysis to avoid significant error. Baseline comparisons between groups were performed using Kruskal-Wallis tests. Within-group comparisons between Pre- and Post- moments were performed using Wilcoxon's signed-rank 


\section{WOMEN}

interventions. Percentage of change ([Post value/Pre value]-1) was calculated and presented for each variable. To report the magnitude of changes the effect size of the point biserial correlation $\left(r_{p b}\right)$ was calculated and converted for standardized Cohen's $d$, categorized as trivial $(d<.2)$, small $(d=.2$ to .6$)$, moderate $(d=.6$ to 1.2$)$, large $(d=1.2$ to 2.0$)$, very large $(d=2.0$ to 4.0$)$ and nearly perfect $(\mathrm{d}>4.0)$. Level of statistical significance for $p<.05$ was assumed. The Statistical Program for Social Sciences - SPSS, version 23.0 for Windows was used.
Results
In total, 78 potential participants were screened for eligibility (see figure 1 flowchart). Firstly, 11 participants were excluded (not meet the inclusion criteria) and 6 participants lost interest after the study intervention was explained. Initially, 61 older adults who met the inclusion criteria were allocated to the different intervention groups. In total 14 participants withdrew and of 2 participants were excluded from analyses due to the low exercise adherence ( $n=1$ for each group). Finally, a total of 47 participants (CAE with $n=19$, CSE with $\mathrm{n}=15$ and CG with $\mathrm{n}=13$ ) completed the 14 -weeks study (see figure 01 ). Table 1 shows the result of the descriptive statistics of the data relating to the characterization of the two experimental and control groups at baseline. There were no statistically significant differences between CAE, CSE and CG for the baseline characteristics or dependent variables at the beginning of the study $(p>.05)$. 


\section{WOMEN}

264

Table 2 shows the differences between the mean and standard deviation values and the degree of significance for each group after 14 weeks of intervention. Significant differences were found for 30'sAC, 8-FUGT and FES in the CAE group $(\mathrm{p}<.05$, moderate effect size), while no significant alterations were observed in these makers for the other groups $(p>.05)$. The backstretch test showed a trend towards improvement, with a moderate effect size, in the CAE group $(\mathrm{p}=.06,+23 \%, d=.85)$. The values for the $30 \mathrm{~s}-\mathrm{CS}$ and instrumental ADL in the CSE group increased significantly $(\mathrm{p}<.05$, with a moderate effect), but other variables remained unchanged after 14 weeks of intervention in this group. Independent ADL scores remained unaffected in all groups $(\mathrm{p}>.05)$.

\section{[Insert figure 2 about here, please]}

Results for the changes in salivary sCORT and sAA after the 14 weeks are shown in the figure 2. Both exercising groups showed a slight increase in $\mathrm{SAA}(\mathrm{CAE}=43 \%, d=.31$ and $\mathrm{CSE}=44 \%, d=.41)$. A small effect size and a non-significant decrease was observed for sAA levels in the CG $(-39 \%, d=.37)$. Salivary CORT levels showed a small increase for all the groups: CAE $(23 \%, d=.22), \operatorname{CSE}(14 \%, d=.53)$ and $\operatorname{CG}(12 \%, d=.43)$.

\section{Discussion}

The most important findings of the present study indicate that the CAE program was proficient in improving upper body strength, agility-dynamic balance and in decreasing the fear of falling. The CSE program also improved lower body strength and instrumental ADL. Independent ADL was not affected. Both exercise groups showed a clinically relevant response by increasing sAA levels. According to a recent study, the concentration levels of 


\section{WOMEN}

cortisol in persons over 80 years old, with the sample collection done at similar times of the day as the ones in our study, varied between 0.30 and $1.1 \mathrm{ug} / \mathrm{mL}$ (Nasr-Esfahani, Moghadam, Tangestaninejad, Mirkhani, \& Momeni, 2006). In this sense, the variation of sCOR after exercise mediation occurred in a range of values that did not affect the participants' state of health. Regarding salivary alpha-amylase, the references values for this age range from 3.1 and $423.1 \mathrm{ug} / \mathrm{mL}$ for adults (Salimetrics UK, 2017).

The sample of this study consisted of 47 institutionalized older women, which presented homogeneity for all the variables and no baseline differences. Adherence was good, with only 2 participants engaging insufficiently. Observed differences between the two active groups (CAE and CSE) and controls were therefore not affected by inherent methodological or selection bias. However, it is important to note that the sample was in a pre-classification state of obesity according to WHO classification $\left(\mathrm{BMI}>25,0 \mathrm{~kg} / \mathrm{m}^{2}\right)$. This is an important factor in our analysis since obesity is not only a risk factor but also interferes with ADL and consequently the independence and autonomy of the older adults (WHO, 2015). This may be why independent ADL was not affected, while instrumental ADL improved with CSE, but not with CAE. Regarding the high scores of CCI present in all groups, the most commonly reported conditions were: peripheral cardiovascular disease, lung and cardiometabolic diseases, followed by mental-health disorders (anxiety and chronic stress). Some of these conditions were treated with beta-blockers that may have interfered with the functioning of the autonomous nervous system and therefore affected our results. It must be kept in mind that this is an older pre-obese population, living in a homecare institution with high dependency needs and physically frail in some cases (Milte \& Crotty, 2014). 


\section{WOMEN}

reduced risk of falls in the older adults when engaging in PE is well documented in the literature and supports our findings (Burton et al., 2015). However, this was only the case for CAE, and not for CSE, in our study. Particularly in our study, this can be explained by the significant improvement in the dynamic balance test in the CAE group $(p \leq .05)$. Looking at the data from the functional fitness tests, some improvements were observed for most tests in both active groups and more so for the CAE group, where the only test not showing improvements was the 30s-CS. Intriguing, this was the only variable which showed a significant increase for the CSE group, where elders could do almost two more repetitions in the $30-\mathrm{CS}$ test in the same 30 seconds. This may be explained by the physiological characteristics of this variable, which recruits more type II fibers and relays more on the anaerobic metabolism. This data is supported by other studies which showed better results in this test when the group engaged in a strength exercise protocol (Daly et al., 2015).

On the other hand, CAE was more effective for all the other variables, including the agility-dynamic balance test. This last variable was the one which improved most after the CAE program, which can also be explained by the aerobic characteristic of this test. Improvements in cardiorespiratory fitness are well documented for older adults that participate in aerobic exercise programs (Theou et al., 2011). For the most optimal improvements, strength and aerobic exercises should be performed, not necessarily together since each type of exercise leads to specific responses and results on physical fitness (ACSM, 2009), but according to the individual needs and limitations of each elderly. The control group, as expected, did not show any improvements in the study variables, instead, a decrease in almost all of the physical fitness tests was observed, emphasizing the importance of being and staying active across the lifespan. Lack of exercise is a risk factor for the loss of independence in older adults (Ensrud et al., 2007). Unlike the present study, most research 


\section{WOMEN}

with sAA is done on the acute effects of exercise (Patacchioli et al., 2015). This study found that sAA levels increased in both active groups, although not reaching statistical significance, but in a range of $43 \%$ for both groups. Moreover, in the control group, sAA levels decreased almost in the same range as the other groups increase $(-39 \%)$. That means not only that exercise is an effective way to elevate sAA, but that these results seem to have clinical relevance. A caveat is that in this older population, effects of medication and illness could have affected outcomes and this needs to be further investigated.

Recent research has shown that exercise has an acute effect on SAA and SCORT, increasing their concentrations after exercise (Hatta, Nishihira, \& Higashiura, 2013; Koibuchi \& Suzuki, 2014). Other studies have linked these biomarkers with other stressful situations, apart from exercise, and have shown much higher responses of sAA and sCORT to stress (Koibuchi \& Suzuki, 2014). Our results showed no statistically significant differences in the concentrations of sAA or sCORT before and after 14 weeks of exercise. However, there is a tendency for an increase in sCORT concentrations for all groups. In the intervention groups, this would be an expected response to exercise, since exercise does not only promote an increase in CORT secretion by the adrenal gland but can also promote an increase in adrenal gland volume itself (Hatta et al., 2013).

The sAA response seems to have a relationship with the exercise, since it shows an increasing trend in both active groups and a decrease in the control group. Although not significant, we can see a tendency of the sAA response to the exercise that could have become significant if the program was extended for a longer period. A study by Hatta et al. (2013) reported an increase in sAA concentrations after a bout of walking exercise. However, it must be considered that this study observed the effect of an acute exercise on sAA, as did most studies investigating this enzyme (Mckune et al., 2014). The same increased tendency 


\section{WOMEN}

of sAA concentrations after 14 weeks of exercise was noticed in our CSE group in accordance to the reported in literature for younger samples (Allgrove et al., 2008). Based on this, and in the tendency shown in a review of 15 articles which studied the effect of different types of exercise on sAA (Koibuchi \& Suzuki, 2014), we can assume that CSE can cause an increase in sAA concentrations, not only immediately after exercise, but also in long-term, which may be associated with the increased in plasma catecholamines seen in response to exercise. Because those hormones are also regulated by the NAS and seem to interfere with salivary secretion they could be involved in the control of salivary electrolytes and protein levels (Chicharro, Lucía, Pérez, Vaquero, \& Ureña, 1998) essential for maintaining mucosal immunity. In addition, saliva composition is mostly regulated by the action of adrenergic mediators, just as catecholamines are. Thus, our results show that sAA seems to be a potential predictor of adrenergic activity, with better accuracy than sCOR.

\section{Conclusion}

In this study, the exercise programs implemented were effective in improving the functional autonomy of older adults. Hormonal responses to exercise showed a tendency to increase. Combining both types of exercise could be used by specialists and other healthcare providers as a feasible exercise program method for the older adult population. Adherence and outcomes of this combination need to be further investigated. It is also possible that a longer intervention protocol could have yielded more significant effects in all the tests used, including the hormonal responses to exercise. Therefore, we recognize the need for new studies that seek to investigate the response of these and other markers, with long-term combined exercise-effects. Also, it is recommended that future studies seek to individually monitor the use of beta-blocker drugs in order to reduce its influence on study results. 
WOMEN

\section{What Does This Article Add?}

This article describes two different exercise protocols aimed at institutionalized populations with a very poor functional fitness level, some even classifying as physically frail. They use a chair for support enabling this population to start exercising from a secure position and gradually improve their fitness levels to a state where the chair is no longer needed. This study also showed that these alternative exercise programs, the Chair elasticband muscle strength exercises (CSE) and the Chair-aerobic exercises to improve walking (CAE), were able to induce relevant changes in the functional fitness levels of the participants where improvements were observed in the Instrumental Activities of Daily Life and the Falls efficacy scale. The trend to increased the levels of salivary alpha-amylase reveal an improvement of mucosal immunity and an involvement in the control of the nervous autonomic system that plays an essential role in regulating blood pressure. The constant search for a better quality of life and autonomy in elderly individuals, is a concern of the social services of health care. The inclusion of these types of exercise can be considered coadjuvant treatment of several diseases that can compromise functional autonomy, and more importantly, showed that chair-based exercises can improve physical fitness, even when started at advanced ages.

ACSM. (2009). American College of Sports Medicine position stand. Progression models in resistance training for healthy adults. Medicine and science in sports and exercise, 
WOMEN

407

408

409

410

411

412

413

414

415

416

417

418

419

420

421

422

423

424

425

426

427

428

41(3), 687-708. https://doi.org/10.1249/MSS.0b013e3181915670

Allgrove, J. E., Gomes, E., Hough, J., \& Gleeson, M. (2008). Effects of exercise intensity on salivary antimicrobial proteins and markers of stress in active men. Journal of sports sciences, 26(6), 653-661. https://doi.org/10.1080/02640410701716790

Burton, E., Cavalheri, V., Adams, R., Browne, C. O., Bovery-spencer, P., Fenton, A. M., ... Hill, K. D. (2015). Effectiveness of exercise programs to reduce falls in older people with dementia living in the community : a systematic review and meta-analysis, $421-$ 434.

Charlson, M., Szatrowski, T. P., Peterson, J., \& Gold, J. (1994). Validation of a combined comorbidity index. Journal of clinical epidemiology, 47(11), 1245-51.

Chen, K., Li, C., Chang, Y., Huang, H., \& Cheng, Y. (2015). An elastic band exercise program for older adults using wheelchairs in Taiwan nursing homes : A cluster randomized trial. International Journal of Nursing Studies, 52(1), 30-38. https://doi.org/10.1016/j.ijnurstu.2014.06.005

Chicharro, J. L., Lucía, A., Pérez, M., Vaquero, A. F., \& Ureña, R. (1998). Saliva composition and exercise. Sports Medicine, 26(1), 17-27. https://doi.org/10.2165/00007256-199826010-00002

Chumlea, W. C., \& Baumgartner, R. N. (1989). Status of anthropometry and body composition data in elderly subjects. The American journal of clinical nutrition, $50(5$ Suppl), 1158-66-5.

Colado, J. C., \& Triplett, N. T. (2008). Effects of a short-term resistance program using elastic bands versus weight machines for sedentary middle-aged women. Journal of 
WOMEN strength and conditioning research / National Strength \& Conditioning Association, 22(5), 1441-8. https://doi.org/10.1519/JSC.0b013e31817ae67a

Daly, R. M., Duckham, R. L., Tait, J. L., Rantalainen, T., Nowson, C. A., Taaffe, D. R., ... Busija, L. (2015). Effectiveness of dual-task functional power training for preventing falls in older people: study protocol for a cluster randomised controlled trial. Trials, 16(1), 120. https://doi.org/10.1186/s13063-015-0652-y

DeSure, A. R., Peterson, K., Gianan, F. V, \& Pang, L. (2013). An exercise program to prevent falls in institutionalized elderly with cognitive deficits: a crossover pilot study. Hawai'i journal of medicine \& public health : a journal of Asia Pacific Medicine \& Public Health, 72(11), 391-5.

Ensrud, K. E., Ewing, S. K., Taylor, B. C., Fink, H. A., Stone, K. L., Cauley, J. A., ... Cawthon, P. M. (2007). Frailty and risk of falls, fracture, and mortality in older women: The study of osteoporotic fractures. Journals of Gerontology - Series A Biological Sciences and Medical Sciences, 62(7), 744-751. https://doi.org/62/7/744 [pii]

Fries, E., Dettenborn, L., \& Kirschbaum, C. (2009). The cortisol awakening response (CAR): Facts and future directions. International Journal of Psychophysiology, 72(1), 67-73. https://doi.org/10.1016/j.ijpsycho.2008.03.014

Furtado, G. E., Uba-Chupel, M., Carvalho, H. M., Souza, N. R., Ferreira, J. P., Teixeira, A. M., ... Smith, B. K. (2016). Effects of a chair-yoga exercises on stress hormone levels, daily life activities, falls and physical fitness in institutionalized older adults. Complementary Therapies in Clinical Practice, 0(0), 144-156. https://doi.org/10.1016/j.ctcp.2016.05.012

Granacher, U., Lacroix, A., Muehlbauer, T., Roettger, K., \& Gollhofer, A. (2013). Effects of 
WOMEN

core instability strength training on trunk muscle strength, spinal mobility, dynamic balance and functional mobility in older adults. Gerontology, 59, 105-113. https://doi.org/10.1159/000343152

Hatta, A., Nishihira, Y., \& Higashiura, T. (2013). Effects of a single bout of walking on psychophysiologic responses and executive function in elderly adults: a pilot study. Clinical interventions in aging, 8, 945-52. https://doi.org/10.2147/CIA.S46405

Koibuchi, E., \& Suzuki, Y. (2014). Exercise upregulates salivary amylase in humans. Experimental and Therapeutic Medicine. https://doi.org/10.3892/etm.2014.1497

Lehninger, A. L., Nelson, D. L., \& Cox, M. M. (2007). Lehninger Princípios de Bioquímica. (Sarvier, Org.) ( $4^{\mathrm{o}}$ ed). São Paulo.

Mckune, A. J., Bach, C. W., Semple, S. J., \& Dyer, B. J. (2014). Salivary cortisol and $\alpha-$ amylase responses to repeated bouts of downhill running. American Journal of Human Biology, 26(6), 850-855. https://doi.org/10.1002/ajhb.22605

Milte, R., \& Crotty, M. (2014). Musculoskeletal health, frailty and functional decline. Best Practice \& Research Clinical Rheumatology, 1-16.

Nasr-Esfahani, M., Moghadam, M., Tangestaninejad, S., Mirkhani, V., \& Momeni, A. R. (2006). Rapid and efficient oxidation of Hantzsch 1,4-dihydropyridines with sodium periodate catalyzed by manganese (III) Schiff base complexes. Bioorganic and Medicinal Chemistry, 14(8), 2720-2724. https://doi.org/10.1016/j.bmc.2005.11.051

Nater, U. M., La Marca, R., Florin, L., Moses, A., Langhans, W., Koller, M. M., \& Ehlert, U. (2006). Stress-induced changes in human salivary alpha-amylase activity -- associations with adrenergic activity. Psychoneuroendocrinology, 31(1), 49-58. 
WOMEN https://doi.org/10.1016/j.psyneuen.2005.05.010

Nater, U. M., \& Rohleder, N. (2009). Salivary alpha-amylase as a non-invasive biomarker for the sympathetic nervous system: Current state of research. Psychoneuroendocrinology, 34(4), 486-496. https://doi.org/10.1016/j.psyneuen.2009.01.014

Nelson, M. E., Rejeski, W. J., Blair, S. N., Duncan, P. W., Judge, J. O., King, A. C., ... Castaneda-Sceppa, C. (2007). Physical activity and public health in older adults: recommendation from the American College of Sports Medicine and the American Heart Association. Medicine and science in sports and exercise, 39(8), 1435-45. https://doi.org/10.1249/mss.0b013e3180616aa2

Patacchioli, F. R., Ghiciuc, C. M., Bernardi, M., Dima-Cozma, L. C., Fattorini, L., Squeo, M. R., ... Perrone, G. (2015). Salivary $\alpha$-amylase and cortisol after exercise in menopause: influence of long-term HRT. Climacteric, 18(4), 528-535. https://doi.org/10.3109/13697137.2015.1008444

Rikli, R. E., \& Jones, C. J. (2013). Development and validation of criterion-referenced clinically relevant fitness standards for maintaining physical independence in later years. Gerontologist, 53(2), 255-267. https://doi.org/10.1093/geront/gns071

Robertson, R. J., Goss, F. L., Rutkowski, J., Lenz, B., Dixon, C., Timmer, J., ... Andreacci, J. (2003). Concurrent validation of the OMNI perceived exertion scale for resistance exercise. Medicine and science in sports and exercise, 35(2), 333-41. https://doi.org/10.1249/01.MSS.0000048831.15016.2A

Robinson, K. R., Leighton, P., Logan, P., Gordon, A. L., Anthony, K., Harwood, R. H., ... Masud, T. (2014). Developing the principles of chair based exercise for older people: a modified Delphi study. BMC geriatrics, 14(1), 65. https://doi.org/10.1186/1471-2318- 
WOMEN

497

498

499

500

501

502

503

504

505

506

507

508

509

510

511

512

513

514

515

516

517

518

14-65

Salimetrics UK. (2017). Salimetrics: Saliva Collection, Saliva EIA kits, Saliva Testing, \& Salivary Bioscience Research.

Sequeira, C. A. da C. (2007). Cuidar de Idosos Dependentes. Coimbra: Quarteto Editora.

Sherrington, C., Fairhall, N., Kirkham, C., Clemson, L., Howard, K., Vogler, C., ... Lord, S. R. (2016). Exercise and fall prevention self-management to reduce mobility-related disability and falls after fall-related lower limb fracture in older people: protocol for the RESTORE (Recovery Exercises and STepping On afteR fracturE) randomised controlled trial. BMC geriatrics, 16(1), 34. https://doi.org/10.1186/s12877-016-0206-5

Spirduso, W. W., Francis, K. L., \& MacRae, P. G. (2005). Physical dimensions of aging. Human Kinetics.

Teixeira, A. M., Ferreira, J. P., Hogervorst, E., Braga, M. F., Bandelow, S., Rama, L., ... Pedrosa, F. M. (2016). Study Protocol on Hormonal Mediation of Exercise on Cognition, Stress and Immunity (PRO-HMECSI): Effects of Different Exercise Programmes in Institutionalized Elders. Frontiers in Public Health, 4, 133. https://doi.org/10.3389/fpubh.2016.00133

Theou, O., Stathokostas, L., Roland, K. P., Jakobi, J. M., Patterson, C., Vandervoort, A. A., \& Jones, G. R. (2011). The effectiveness of exercise interventions for the management of frailty: a systematic review. Journal of aging research, 2011, 569194. https://doi.org/10.4061/2011/569194

Tinetti, M. E., Richman, D., \& Powell, L. (1990). Falls efficacy as a measure of fear of falling. Journal of gerontology, 45(6), P239-P243. 
WOMEN https://doi.org/10.1093/geronj/45.6.P239

520

Wallace, M., \& Shelkey, M. (2007). Katz Index of Independence in Activities of Daily Living (ADL). Urologic nursing : official journal of the American Urological Association Allied, 27(1), 93-94. https://doi.org/10.1097/00004045-200105000-00020

Walsh, N. P., Gleeson, M. M., Shephard, R. J., Jeffrey, M. G., Woods, A., Bishop, N. C., ... Simon, P. (2011). Part one : Immune function and exercise. Exercise immunology review, 17, 6-63.

WHO. (2015). World report on ageing and health. Who. https://doi.org/10.1007/s13398-014$0173-7.2$ 
Table 1. Characteristics of experimental and control groups at baseline and comparison between groups by ANOVA one-way.

\begin{tabular}{|c|c|c|c|c|c|}
\hline & $\begin{array}{l}\text { Chair-aerobic improve } \\
\text { walk exercises }(\mathrm{n}=19)\end{array}$ & $\begin{array}{l}\text { Chair elastic-band strength } \\
\text { exercises }(n=15)\end{array}$ & $\begin{array}{l}\text { Control group non } \\
\text { exercise }(n=13)\end{array}$ & \multirow[t]{2}{*}{ f value } & \multirow{2}{*}{$\mathrm{p}$ value } \\
\hline & mean $(\mathrm{SD})$ & mean $(\mathrm{SD})$ & mean $(\mathrm{SD})$ & & \\
\hline Chronological age (years) & $79.00 \pm 7.63$ & $83.47 \pm 4.92$ & $80.85 \pm 10.86$ & 1.314 & .279 \\
\hline Height (centimeters) & $1.50 \pm .40$ & $1.47 \pm .41$ & $1.51 \pm .71$ & 2.374 & .105 \\
\hline Weight (kilos) & $61.47 \pm 1.039$ & $63.97 \pm 15.93$ & $69.91 \pm 16.00$ & 1.431 & .250 \\
\hline Body mass index (score) & $27.09 \pm 4.13$ & $29.51 \pm 7.24$ & $30.73 \pm 6.43$ & 1.595 & .214 \\
\hline Charlson Comorbidity Index (score) & $7.21 \pm 1.88$ & $8.07 \pm 1.91$ & $8.77 \pm 2.68$ & 2.114 & .133 \\
\hline Medication use (per unit) & $3.12(.88)$ & $3.01(.34)$ & $4.02(1.08)$ & 1.567 & .234 \\
\hline
\end{tabular}


Table 2. Comparison between pre and post exercise intervention and control group (no exercise intervention)

Aerobic improve walking (n=19)

Pre Post

\begin{tabular}{lccc} 
& Pre & Post & Percent \\
& Mean (SD) & Mean (SD) & change (\%) \\
\cline { 1 - 3 } & & & +43 \\
Alpha amylase (ug/mL) & $60.33 \pm 126.55$ & $86.50 \pm 151.22$ & +13 \\
Cortisol (ug/mL) & $.27 \pm .07$ & $.31 \pm .29$ & +23 \\
Back stretch test $(\mathrm{cm})^{\#}$ & $27.05 \pm 12.75$ & $33.16 \pm 12.92$ & +23 \\
30 seconds Arm-curl test (reps) & $8.79 \pm 3.12$ & $11.63 \pm 3.67^{* *}$ & +32 \\
30 seconds chair-stand test (reps) & $9.26 \pm 3.01$ & $10.05 \pm 3.01$ & +9 \\
Eight foot-up and-go test (time) & $12.77 \pm 4.89$ & $9.89 \pm 2.53^{* *}$ & -23 \\
Falls efficacy scale (score) & $54.00 \pm 26.76$ & $25.53 \pm 18.66^{* *}$ & -53 \\
Instrumental ADL (score) & $20.53 \pm 5.87$ & $18.58 \pm 7.14$ & -9 \\
Independent ADL (score) $^{\&}$ & $5.16 \pm 1.42$ & $5.11 \pm 1.24$ & -1 \\
\hline
\end{tabular}

Elastic-band strength exercises $(n=15)$

Effect

\begin{tabular}{cc} 
Pre & Post \\
\hline Mean & Mean \\
(SD) & (SD)
\end{tabular}

Percent

change

(SD)

$31.19 \pm 52.15 \quad 44.66 \pm 102.91$

$.24 \pm .12$

$.27 \pm .11$

$27.77 \pm 10.17$

$11.20 \pm 5.80$

$29.23 \pm 13.09$

$6.67 \pm 2.55$

$11.93 \pm 3.79$

$8.47 \pm 3.44 * *$

$20.62 \pm 10.30$

$15.52 \pm 6.82$

$42.73 \pm 23.73$

$23.73 \pm 4.96$

$31.4 \pm 16.27$

$26.73 \pm 4.17 * *$

$4.87 \pm 1.12$

$4.53 \pm 1.64$

+44
+14
+5
+7
+27
-25
-27
+13
-7

Effect

Size (d)

.41

.53

.53
.09

.74

1.12

1.12

.63

.92

.43
Control group non exercise (n=13)

\begin{tabular}{cccc} 
Pre & Post & $\begin{array}{c}\text { Percent } \\
\text { Change (\%) }\end{array}$ & $\begin{array}{c}\text { Effect } \\
\text { Size (d) }\end{array}$ \\
\cline { 1 - 2 } Mean (SD) & Mean (SD) & & \\
\hline $72.45 \pm 154.10$ & $44.21 \pm 73.19$ & -39 & .37 \\
$.26 \pm .11$ & $0.30 \pm .14$ & +12 & .43 \\
$25.88 \pm 13.12$ & $28.85 \pm 13.26$ & +11 & .46 \\
$8.08 \pm 4.57$ & $7.54 \pm 3.26$ & -7 & .36 \\
$7.15 \pm 2.97$ & $6.85 \pm 3.46$ & -4 & .16 \\
$18.16 \pm 12.50$ & $16.72 \pm 7.87$ & -8 & .09 \\
$39.54 \pm 20.55$ & $27.92 \pm 15.30$ & -29 & .68 \\
$21.77 \pm 5.31$ & $22.62 \pm 4.56$ & 4 & .21 \\
$4.46 \pm 1.33$ & $4.62 \pm .96$ & 4 & .32
\end{tabular}

${ }^{* *} p<.01 ;{ }^{*} p<.05,{ }^{*}$ Comparison using T-test for paired samples; ${ }^{\&}$ Compared using Wilcoxon-signed rank test. 